\title{
ON THE SET OF SUBSEQUENTIAL LIMIT POINTS OF SUCCESSIVE APPROXIMATIONS( $\left.{ }^{(}\right)$
}

\author{
BY \\ J. B. DIAZ AND F. T. $\operatorname{METCALF}\left({ }^{2}\right)$
}

1. Introduction. Let $A: S \rightarrow S$ be continuous, where $S$ is a nonempty metric space. Given $x \in S$, it is natural to ask under what circumstances the (Picard) sequence of iterates $\left\{A^{m}(x)\right\}_{m=1}^{\infty}$ converges to a fixed point of $A$. An answer to this question is given, for example, by the well-known "Banach's contraction mapping principle" [1]. This principle states that if $S$ is a complete metric space and $A$ is "strictly" a contraction over $S$, then, for any $x \in S$, the sequence of iterates $\left\{A^{m}(x)\right\}_{m=1}^{\infty}$ converges to a unique fixed point of $A$. More recent answers to this same general question have been given by Edelstein [2], [3] and Browder and Petryshyn [4]; [5].

The ultimate objective of the present investigation is also to give an answer to the same general question about the convergence of the sequence of iterates. Generally speaking, the sequence of iterates may contain no convergent subsequence or, on the other hand, it may contain many convergent subsequences (with distinct limits). The approach to be followed here is to study first the structure of the set of subsequential limit points of the sequence of iterates, on the basis of hypotheses which do not guarantee the convergence of the entire sequence of iterates. The final aim is, of course, to determine conditions under which the sequence actually converges; but the results obtained concerning the structure of the set of subsequential limit points appear to be of interest in themselves.

This paper had its origin in the stimulating series of lectures delivered by Professor F. Tricomi at the Institute for Fluid Dynamics and Applied Mathematics, University of Maryland, in April of 1966, see [6]. In the course of his lectures Professor Tricomi drew our attention to his first published paper, [7], which is concerned with the iteration of a real-valued function. Tricomi's result (which makes no mention of derivatives) contains, as a special case, a classical convergence criterion for iterations (which is based on assuming that a certain derivative is less than one in absolute value; see, for example, Ostrowski [8, pp. 26-27]). §2 deals

Presented to the Society, January 24, 1967 under the title On the structure of the set of limit points of successive approximations; received by the editors May 30, 1967 and, in revised form, September 20, 1967.

(1) Abstract, Notices Amer. Math. Soc. 14 (1967), p. 107; research announcement, Bull. Amer. Math. Soc. 73 (1967), 516-519.

$\left(^{2}\right)$ The research of the authors was supported in part by the Air Force Office of Scientific Research, Grant AFOSR 1122-66. 
with the above mentioned result of Tricomi for a real-valued function (Theorem 1) and a generalization to a possibly discontinuous function (Theorem 1.1).

$\$ 3$ is concerned with the structure of the set of subsequential limit points of the sequence of iterates $\left\{A^{m}(x)\right\}_{m=1}^{\infty}$, where $A: S \rightarrow S$ is continuous and $S$ is a nonempty metric space. The main result is Theorem 2 , which, under suitable hypotheses, asserts that the set of subsequential limit points of the sequence of iterates is a closed and connected subset of the set of fixed points of $A$.

$\S 4$ contains a variation of Theorem 2 , namely Theorem 3 , whose conclusion states that either the sequence of iterates $\left\{A^{m}(x)\right\}_{m=1}^{\infty}$ contains no convergent subsequence, or $\lim _{m \rightarrow \infty} A^{m}(x)$ exists and is a fixed point of $A$.

$\$ 5$ is devoted to applications of the preceding results (in particular, Theorem 3 ). Theorem 4 is related to the results of Edelstein [3] and Krasnosel'skir [9]; while Theorem 5 is connected with the work of Fridman [10].

Finally, $\$ 6$ contains some further modifications of Theorems 2 and 3, involving the additional hypothesis of "asymptotic regularity" of Browder and Petryshyn [5, p. 572, Definition 1].

2. The result of Tricomi. In [7, p. 2, Theorem], Tricomi obtains a general result concerning iterations, a special case of which [7, p. 4, Corollary] may. be formulated as follows:

THEOREM 1. Let $A$ be a real-valued continuous function on the (finite or infinite) open interval $a<x<b$, whose values lie in the same interval. Suppose

(i) there exists a number $p$, with $a<p<b$, such that $A(p)=p$;

(ii) $|A(x)-p|<|x-p|$ for $a<x<b, x \neq p$. Then, for every $a<x<b$,

$$
\lim _{m \rightarrow \infty} A^{m}(x)=p
$$

where $A^{m}$ denotes the mth iterate of the function $A$.

Proof. Let $x$ be given such that $a<x<b$. If $x=p$, or if $A^{m}(x)=p$ for some integer $m \geqq 1$, then the desired assertion is obviously true. Suppose, therefore, that $A^{m}(x) \neq p$ for every $m=0,1,2, \ldots$ Then the sequence of positive numbers $\left\{\left|A^{m}(x)-p\right|\right\}_{m=1}^{\infty}$ is a decreasing sequence, because, from (ii),

$$
\left|A^{m+1}(x)-p\right|=\left|A\left(A^{m}(x)\right)-p\right|<\left|A^{m}(x)-p\right|
$$

for $m=1,2, \ldots ;$ hence,

$$
\lim _{m \rightarrow \infty}\left|A^{m}(x)-p\right|=L \geqq 0 .
$$

If $L=0$, then there is nothing left to prove. Suppose, therefore, that $L>0$. Then, either there exists a decreasing subsequence $\left\{A^{m_{i}}(x)-p\right\}_{i=1}^{\infty}$ tending to $+L$, or an increasing sequence $\left\{A^{m_{i}}(x)-p\right\}_{i=1}^{\infty}$ tending to $-L$. Consider the first case, since the argument is similar when the limit is $-L$. Therefore, suppose that

$$
\lim _{i \rightarrow \infty}\left[A^{m_{i}}(x)-p\right]=\lim _{i \rightarrow \infty} A^{m_{i}}(x)-p=L,
$$


and that $A^{m_{i}}(x)-p>L$ for $i=1,2, \ldots$ It is clear from the last inequality that

$$
a<p<p+L<A^{m_{i}}(x)<b, \quad i=1,2, \ldots,
$$

so that the function $A$ is defined at $p+L$. Since $\lim _{i \rightarrow \infty} A^{m_{i}}(x)=p+L$ and $A$ is continuous, one has that

$$
\lim _{i \rightarrow \infty} A^{m_{i}+1}(x)=A\left(\lim _{i \rightarrow \infty} A^{m_{i}}(x)\right)=A(p+L) .
$$

Hence, using (ii),

$$
\begin{aligned}
L & =\lim _{i \rightarrow \infty}\left|A^{m_{i}+1}(x)-p\right|=\left|\lim _{i \rightarrow \infty} A^{m_{i}+1}(x)-p\right| \\
& =|A(p+L)-p|<|(p+L)-p|=L,
\end{aligned}
$$

a contradiction. Thus, $L$ must be zero, and the proof is complete.

Tricomi [7, p. 2 and p. 6 (Osservazione)] remarks that his result is valid regardless of the existence of the derivative of $A$, or even the continuity of $A$. Along these lines, a seemingly stronger version of Theorem 1 holds, in which $A$ is not required to be continuous, but a higher order iterate $A^{k}$ is required to be continuous (as will be seen from the proof, this theorem amounts to $k$ applications of Theorem 1).

THEOREM 1.1. Let $A$ be a real-calued function on the (finite or infinite) open interval $a<x<b$, whose values lie in the same interval; and such that $A^{k}$ is continuous, where $k$ is a positive integer. Suppose

(i) there exists a number $p$, with $a<p<b$, such that $A(p)=p$;

(ii) $|A(x)-p|<|x-p|$ for $a<x<p, x \neq p$. Then, for every $a<x<b$,

$$
\lim _{m \rightarrow \infty} A^{m}(x)=p,
$$

where $A^{m}$. denotes the mth iterate of the function $A$.

Proof. If $k=1$, then the present theorem reduces to Theorem 1. Suppose, for definiteness, that $k \geqq 2$. It will now be shown that the hypotheses of Theorem 1 hold for the continuous function $A^{k}$. Clearly, $A^{k}$ takes the interval $a<x<b$ into itself, and $A^{k}(p)=p$. Hypothesis (ii) of Theorem 1.1 implies

$$
\left|A^{k}(x)-p\right| \leqq\left|A^{k-1}(x)-p\right| \leqq \cdots \leqq|A(x)-p|<|x-p|
$$

whenever $a<x<b$ and $x \neq p$. Since the last inequality is strict, one has that $\left|A^{k}(x)-p\right|<|x-p|$ whenever $a<x<b$ and $x \neq p$.

Let $x$ be such that $a<x<b$. Then Theorem 1, applied to the continuous function $A^{k}$, gives that $\lim _{m \rightarrow \infty} A^{m k}(x)=p$. Now, replacing $x$ by $A(x), A^{2}(x), \ldots, A^{k-1}(x)$, in succession, in the last equation, one obtains that

$$
\lim _{m \rightarrow \infty} A^{m k+j}(x)=p, \quad j=0,1, \ldots, k-1 .
$$

These last $k$ equations give $\lim _{m \rightarrow \infty} A^{m}(x)=p$, as desired.

REMARK 1. The requirement of the continuity of $A$ can be removed altogether if (ii) is replaced with the stronger condition 
(ii') there exists a number $\eta$, with $0<\eta<1$, such that $|A(x)-p| \leqq \eta|x-p|$ for $a<x<b, x \neq p$.

Clearly, this implies that the number $L$, which appears in the proof of Theorem 1 , must be zero.

REMARK 2. Theorem 1 remains valid, with similar reasoning, when the open interval $a<x<b$ is replaced by a closed interval $a \leqq x \leqq b$, or even by a half-closed interval (either $a \leqq x<b$ or $a<x \leqq b$ ). As a matter of fact, the interval $a \leqq x \leqq b$ may be replaced by any closed set $S$ of real numbers. The only modification in the argument occurs in showing that the number $p+L$ belongs to $S$ (which, in the case of the interval $a<x<b$, was done by means of the inequalities $a<p<p+L<$ $\left.A^{m_{1}}(x)<b\right)$. In the case of a set $S$, one sees that $p+L$ belongs to $S$ because $A$ takes $S$ into $S, \lim _{i \rightarrow \infty} A^{m_{1}}(x)=p+L$, and $S$ is closed. It is in this last form, involving a closed set $S$, that a generalization of Theorem 1 will be given in $\S 3$.

REMARK 3. The previous theorems have been concerned with a single fixed point $p$. It is natural to ask whether similar theorems hold when the set of fixed points, call it $F(A)$, may consist of more than one point. It seems reasonable, in these circumstances, to replace condition (ii) by

$$
\inf _{p \in F(A)}|A(x)-p|<\inf _{p \in F(A)}|x-p|
$$

for $a<x<b, x \notin F(A)$, which clearly reduces to (ii) when $F(A)$ consists of exactly one point. It is this condition which will be employed in Theorem 2 of $\S 3$, which can be thought of as a generalization of Theorem 1. However, if one replaces condition (ii) of Theorem 1.1 with condition (ii*), then the resulting proposition need not be true when $F(A)$ consists of more than one point, as the following example shows.

Define $A$ on the interval $-2<x<2$ as follows:

$$
\begin{array}{rlrl}
A(x) & =\frac{1}{2}(1-x), & -2 & <x<-1, \\
& =x, & -1 \leqq x \leqq+1, \\
& =-\frac{1}{2}(1+x), & 1 & <x<2 .
\end{array}
$$

The function $A$ takes the interval $-2<x<2$ into itself and is not continuous. On the other hand, the function $A^{2}$ :

$$
\begin{array}{rlrl}
A^{2}(x) & =-\frac{3}{4}+\frac{1}{4} x, & -2 & <x<-1, \\
& =x, & -1 \leqq x \leqq 1, \\
& =\frac{3}{4}+\frac{1}{4} x, & 1 & <x<2,
\end{array}
$$

is continuous. The set $F(A)$ is the interval $-1 \leqq x \leqq 1$. If $1<x<2$, then

and

$$
\begin{aligned}
\inf _{-1 \leqq p \leqq 1}|A(x)-p| & =\inf _{-1 \leqq p \leqq 1}\left|-\frac{1}{2}(1-x)-p\right| \\
& =\left|-\frac{1}{2}(1-x)+1\right|=\frac{1}{2}(x-1)
\end{aligned}
$$$$
\inf _{-1 \leqq p \leqq 1}|x-p|=x-1 \text {; }
$$ 
while, if $-2<x<-1$, then

and

$$
\begin{aligned}
\inf _{-1 \leqq p \leqq 1}|A(x)-p| & =\inf _{-1 \leqq p \leqq 1}\left|\frac{1}{2}(1-x)-p\right| \\
& =\left|\frac{1}{2}(1-x)-1\right|=\frac{1}{2}(-x-1)
\end{aligned}
$$

$$
\inf _{-1 \leqq p \leqq 1}|x-p|=-x-1 ;
$$

hence (ii*) holds. If $-2<x<-1$, it can be verified by mathematical induction that

and

$$
A^{2 i-1}(x)=\left(1-\frac{1}{2^{2 i-1}}\right)-\frac{1}{2^{2 i-1}} x
$$

for $i=1,2, \ldots$ Since

$$
A^{2 i}(x)=-\left(1-\frac{1}{2^{2 i}}\right)+\frac{1}{2^{2 i}} x
$$

$$
\lim _{i \rightarrow \infty} A^{2 i-1}(x)=1 \text { and } \lim _{i \rightarrow \infty} A^{2 i}(x)=-1,
$$

the sequence of iterates $\left\{A^{m}(x)\right\}_{m=1}^{\infty}$ does not converge.

3. The main result. Let $A: S \rightarrow S$, where $S$ is a nonempty metric space. For brevity, the following notation will be employed throughout the sequel. Let $F(A)$ denote the set of fixed points of $A$, that is, $F(A)=\{x \in S \mid A(x)=x\}$. Also, $d(x, F(A))$ will denote the distance between the point $x \in S$ and the set $F(A)$, that is,

$$
d(x, F(A))=\inf _{p \in F(A)} d(x, p) .
$$

Further, for $x \in S, \mathscr{L}(x)$ will denote the set of subsequential limit points of the sequence of iterates $\left\{A^{m}(x)\right\}_{m=1}^{\infty}$, that is, $\mathscr{L}(x)$ is the set of all $y \in S$ such that $y=\lim _{i \rightarrow \infty} A^{m_{i}}(x)$ for some subsequence $\left\{A^{m}(x)\right\}_{i=1}^{\infty}$ of the sequence $\left\{A^{m}(x)\right\}_{m=1}^{\infty}$. As usual, a subset $K$ of $S$ will be said to be compact if every sequence of points from $K$ contains a subsequence which converges to a point in $S$.

THEOREM 2. Let $A: S \rightarrow S$ be continuous. Suppose

(i) $F(A)$ is nonempty and compact;

(ii) for each $x \in S$, with $x \notin F(A)$, one has

$$
d(A(x), F(A))<d(x, F(A)) .
$$

Then, for $x \in S$, the set $\mathscr{L}(x)$ is a closed and connected subset of $F(A)$. Either $\mathscr{L}(x)$ is empty, or it contains exactly one point, or it contains uncountably many points. In case $\mathscr{L}(x)$ is just one point, then $\lim _{m \rightarrow \infty} A^{m}(x)$ exists and belongs to $F(A)$. In case $\mathscr{L}(x)$ is uncountable, then it is contained in the boundary of $F(A)$.

Proof. 1. If $\mathscr{L}(x)$ is empty, then there is nothing left to prove. Therefore, throughout the rest of the argument it will be supposed that $\mathscr{L}(x)$ is nonempty. If $x \in F(A)$, or if $A^{k}(x) \in F(A)$ for some integer $k \geqq 1$, then $\lim _{m \rightarrow \infty} A^{m}(x)$ exists and 
belongs to $F(A)$, and the theorem is true. Therefore, it will also be supposed throughout the remainder of the argument that $A^{m}(x) \notin F(A)$ for every $m=0,1$, $2, \ldots$ Then, the sequence of positive numbers $\left\{d\left(A^{m}(x), F(A)\right)\right\}_{m=1}^{\infty}$ is a decreasing sequence, because, from (ii),

$$
d\left(A^{m+1}(x), F(A)\right)=d\left(A\left(A^{m}(x)\right), F(A)\right)<d\left(A^{m}(x), F(A)\right)
$$

for $m=1,2, \ldots$; hence, $\lim _{m \rightarrow \infty} d\left(A^{m}(x), F(A)\right)$ exists and is nonnegative.

2. $\mathscr{L}(x)$ is a subset of $F(A)$. Let $\left\{A^{m_{i}}(x)\right\}_{i=1}^{\infty}$ be a convergent subsequence of $\left\{A^{m}(x)\right\}_{m=1}^{\infty}$. If it were true that $\lim _{i \rightarrow \infty} A^{m_{i}}(x) \notin F(A)$, then one would have that, by the continuity of the distance function $d$, the continuity of $A$, and (ii),

$$
\begin{aligned}
\lim _{i \rightarrow \infty} d\left(A^{m_{i}+1}(x), F(A)\right) & =d\left(\lim _{i \rightarrow \infty} A\left(A^{m_{i}}(x)\right), F(A)\right) \\
& =d\left(A\left(\lim _{i \rightarrow \infty} A^{m_{i}}(x)\right), F(A)\right) \\
& <d\left(\lim _{i \rightarrow \infty} A^{m_{i}}(x), F(A)\right)=\lim _{i \rightarrow \infty} d\left(A^{m_{i}}(x), F(A)\right),
\end{aligned}
$$

which is a contradiction, since $\lim _{m \rightarrow \infty} d\left(A^{m}(x), F(A)\right)$ exists. Consequently, $\lim _{i \rightarrow \infty} A^{m_{i}}(x) \in F(A)$, and hence $\mathscr{L}(x) \subseteq F(A)$. Notice also that, since $\lim _{i \rightarrow \infty} A^{m_{i}}(x)$ $\in F(A)$, it follows that

$$
\lim _{i \rightarrow \infty} d\left(A^{m_{i}}(x), F(A)\right)=d\left(\lim _{i \rightarrow \infty} A^{m_{i}}(x), F(A)\right)=0 ;
$$

this means that the sequence $\left\{d\left(A^{m}(x), F(A)\right)\right\}_{m=1}^{\infty}$ decreases to zero.

3. $\mathscr{L}(x)$ is closed. All that this amounts to is that "a limit point of limit points is also a limit point". Suppose that $\left\{y_{i}\right\}_{i=1}^{\infty}$ is a convergent sequence of points of $\mathscr{L}(x)$, with $y_{i}=\lim _{j \rightarrow \infty} A^{m_{i j}}(x)$, for $i=1,2, \ldots$, converging to a point $y \in F(A)$. It has to be shown that $y$ actually belongs to $\mathscr{L}(x)$.

Let $\varepsilon>0$. There exist positive integers $J(\varepsilon, i)(i=1,2, \ldots)$, such that

$$
d\left(y_{1}, A^{m_{i j}}(x)\right)<\frac{1}{2} \varepsilon, \quad j \geqq J(\varepsilon, i), \quad i=1,2, \ldots
$$

Define the sequence of numbers $\{k(i)\}_{i=1}^{\infty}$ by recursion, where $k(1)=J(\varepsilon, 1)$, and

$$
k(i+1)=\min \left\{j \mid J(\varepsilon, i+1) \leqq j<\infty, m_{i, k(i)}<m_{i+1, j}\right\}
$$

for $i=1,2, \ldots$ Upon setting $n_{i}=m_{i, k:(i)}, i=1,2, \ldots$, one has that the sequence of numbers $\left\{n_{i}\right\}_{i=1}^{\infty}$ is an increasing sequence. Also, there exists a positive integer $I(\varepsilon)$ such that $d\left(y, y_{i}\right)<\frac{1}{2} \varepsilon$ whenever $i \geqq I(\varepsilon)$. Then the subsequence of iterates $\left\{A^{n}(x)\right\}_{i=1}^{\infty}$ converges to $y$, because, for all $i \geqq I(\varepsilon)$,

$$
d\left(y, A^{n_{i}}(x)\right) \leqq d\left(y, y_{i}\right)+d\left(y_{i}, A^{n_{i}}(x)\right)<\frac{1}{2} \varepsilon+\frac{1}{2} \varepsilon=\varepsilon .
$$

This means that $y$ belongs to $\mathscr{L}(x)$.

4. $\mathscr{L}(x)$ is connected. Suppose, contrary to what one wishes to prove, that the closed set $\mathscr{L}(x)$ is not connected. Then $\mathscr{L}(x)=S_{1} \cup S_{2}$, where $S_{1} \cap S_{2}$ is empty, 
and both $S_{1}$ and $S_{2}$ are closed and nonempty. Since $\mathscr{L}(x) \subseteq F(A)$ and $F(A)$ is compact, one has that

$$
d\left(S_{1}, S_{2}\right)=\inf _{y \in S_{1}: z \in S_{2}} d(y, z)>0
$$

because $S_{1}$ and $S_{2}$ are closed, compact, and disjoint. Let

and

$$
S_{1}^{+}=\left\{y \in F(A) \mid d\left(y, S_{1}\right) \leqq \frac{1}{4} d\left(S_{1}, S_{2}\right)\right\}
$$

$$
S_{2}^{+}=\left\{y \in F(A) \mid d\left(y, S_{2}\right) \leqq \frac{1}{4} d\left(S_{1}, S_{2}\right)\right\} .
$$

The closed and compact sets $S_{1}^{+}$and $S_{2}^{+}$have no points in common, for if $y \in$ $S_{1}^{+} \cap S_{2}^{+}$, then one would have

$$
0<d\left(S_{1}, S_{2}\right) \leqq d\left(S_{1}, y\right)+d\left(y, S_{2}\right) \leqq \frac{1}{2} d\left(S_{1}, S_{2}\right),
$$

a contradiction. Therefore $d\left(S_{1}^{+}, S_{2}^{+}\right)>0$.

Since the function $A$ is continuous on the closed and compact set $F(A)$, it has a property that may be described as "being uniformly continuous with respect to $F(A)$ on the set $R=F(A) \cup\left\{z \mid z=A^{m}(x), 1 \leqq m<\infty\right\}$ ". (The set $R$, it is to be noticed, need not be compact.) More precisely, for every $\varepsilon>0$, there exists $\delta(\varepsilon)>0$ such that, whenever $y \in F(A)$ and $z \in R$, with $d(y, z)<\delta(\varepsilon)$, then one has

$$
d(A(y), A(z))<\varepsilon
$$

This property of $A$ can be proved by contradiction, as follows. For, if this property did not hold, then there would exist $\varepsilon_{0}>0$ and sequences of points $\left\{y_{m}\right\}_{m=1}^{\infty}$ and $\left\{z_{m}\right\}_{m=1}^{\infty}$, with $y_{m} \in F(A)$ and $z_{m} \in R$, such that both $\lim _{m \rightarrow \infty} d\left(y_{m}, z_{m}\right)=0$ and $d\left(A\left(y_{m}\right), A\left(z_{m}\right)\right) \geqq \varepsilon_{0}>0$ for $m=1,2, \ldots$ Since $F(A)$ is closed and compact, one may suppose, without loss (by passing to a subsequence, and then relabeling if necessary), that the sequence $\left\{y_{m}\right\}_{m=1}^{\infty}$ actually converges to a point $y \in F(A)$. From the triangle inequality one has that

$$
d\left(y, z_{m}\right) \leqq d\left(y, y_{m}\right)+d\left(y_{m}, z_{m}\right), \quad m=1,2, \ldots,
$$

which means that $\lim _{m \rightarrow \infty} d\left(y, z_{m}\right)=0$. Again, from the triangle inequality, one has that

$$
\begin{aligned}
d\left(A\left(y_{m}\right), A\left(z_{m}\right)\right) & \leqq d\left(A\left(y_{m}\right), y\right)+d\left(y, A\left(z_{m}\right)\right) \\
& =d\left(y_{m}, y\right)+d\left(A(y), A\left(z_{m}\right)\right),
\end{aligned}
$$

for $m=1,2, \ldots$ However, in view of $\lim _{m \rightarrow \infty} d\left(y, z_{m}\right)=0$ and the continuity of $A$ at $y$, the last inequality implies that

$$
\lim _{m \rightarrow \infty} d\left(A\left(y_{m}\right), A\left(z_{m}\right)\right)=0,
$$

which contradicts $d\left(A\left(y_{m}\right), A\left(z_{m}\right)\right) \geqq \varepsilon_{0}>0$.

Since $F(A)$ is closed and compact, there exist points $p_{m} \in F(A)$ such that

$$
d\left(A^{m}(x), F(A)\right)=d\left(A^{m}(x), p_{m}\right)
$$


for $m=1,2, \ldots$ Further, by (ii) and part 2 of the proof, the sequence $\left\{d\left(A^{m}(x), p_{m}\right)\right\}_{m=1}^{\infty}$ decreases to zero. Choose $\varepsilon>0$ and let the positive integer $M=M(\varepsilon)$ be such that

$$
d\left(A^{m}(x), p_{m}\right)<\min \left(\frac{1}{2} \varepsilon, \delta\left(\frac{1}{2} \varepsilon\right)\right),
$$

for all $m \geqq M$, where $\delta$ arises from the "uniformity property" of $A$ on the set $R$. Then,

$$
\begin{aligned}
d\left(A^{m}(x), A^{m+1}(x)\right) & \leqq d\left(A^{m}(x), p_{m}\right)+d\left(A^{m+1}(x), p_{m}\right) \\
& =d\left(A^{m}(x), p_{m}\right)+d\left(A\left(A^{m}(x)\right), A\left(p_{m}\right)\right) \\
& <\frac{1}{2} \varepsilon+\frac{1}{2} \varepsilon=\varepsilon
\end{aligned}
$$

for all $m \geqq M$. It has thus been shown that

$$
\lim _{m \rightarrow \infty} d\left(A^{m}(x), A^{m+1}(x)\right)=0 .
$$

It will next be shown, by contradiction, that

$$
\lim _{m \rightarrow \infty} d\left(A^{m}(x), S_{1}^{+} \cup S_{2}^{+}\right)=0 .
$$

For, suppose that this limit were not zero; then there would exist $\varepsilon>0$ and a subsequence of iterates $\left\{A^{m_{i}}(x)\right\}_{i=1}^{\infty}$ such that

$$
d\left(A^{m_{i}}(x), S_{1}^{+} \cup S_{2}^{+}\right) \geqq \varepsilon>0
$$

for all $i=1,2, \ldots$ Consider the corresponding subsequence of points in $F(A)$, $\left\{p_{m_{i}}\right\}_{i=1}^{\infty}$, such that $d\left(A^{m_{i}}(x), F(A)\right)=d\left(A^{m_{i}}(x), p_{m_{i}}\right)$ for $i=1,2, \ldots$ In view of (ii) and part 2 of the proof, one has that the sequence $\left\{d\left(A^{m_{i}}(x), p_{m_{1}}\right)\right\}_{i=1}^{\infty}$ decreases to zero. Since $F(A)$ is closed and compact, one may suppose, without loss, that the subsequence $\left\{p_{m_{i}}\right\}_{i=1}^{\infty}$ actually converges to a point $p \in F(A)$. From the triangle inequality

$$
d\left(A^{m_{\mathfrak{i}}}(x), p\right) \leqq d\left(A^{m_{\mathfrak{i}}}(x), p_{m_{\mathfrak{i}}}\right)+d\left(p_{m_{\mathfrak{i}}}, p\right), \quad i=1,2, \ldots,
$$

one has that $\lim _{i \rightarrow \infty} d\left(A^{m_{i}}(x), p\right)=0$, so that $p$ actually belongs to $\mathscr{L}(x)$. However, since

$$
p \in \mathscr{L}(x)=S_{1} \cup S_{2} \subseteq S_{1}^{+} \cup S_{2}^{+},
$$

this contradicts the initial hypothesis that $d\left(A^{m_{i}}(x), S_{1}^{+} \cup S_{2}^{+}\right) \geqq \varepsilon>0$ for all $i=1,2, \ldots$.

Thus, it has been shown that both

and

$$
\lim _{m \rightarrow \infty} d\left(A^{m}(x), A^{m+1}(x)\right)=0
$$

$$
\lim _{m \rightarrow \infty} d\left(A^{m}(x), S_{1}^{+} \cup S_{2}^{+}\right)=0 .
$$

Therefore, there exists a positive integer $M$ such that, for all $m \geqq M$, one has both

and

$$
d\left(A^{m}(x), A^{m+1}(x)\right)<\frac{1}{3} d\left(S_{1}^{+}, S_{2}^{+}\right)
$$

$$
d\left(A^{m}(x), S_{1}^{+} \cup S_{2}^{+}\right)<\frac{1}{3} d\left(S_{1}^{+}, S_{2}^{+}\right) \text {. }
$$


From this last inequality, for any $m \geqq M$, it follows that one has either

$$
d\left(A^{m}(x), S_{1}^{+}\right)<\frac{1}{3} d\left(S_{1}^{+}, S_{2}^{+}\right)
$$

or

$$
d\left(A^{m}(x), S_{2}^{+}\right)<\frac{1}{3} d\left(S_{1}^{+}, S_{2}^{+}\right) .
$$

The set of positive integers $m \geqq M$, such that $d\left(A^{m}(x), S_{1}^{+}\right)<\frac{1}{3} d\left(S_{1}^{+}, S_{2}^{+}\right)$, is not empty, because $S_{1}^{+}$contains at least one limit point of the set of iterates $\left\{A^{i}(x)\right\}_{i=1}^{\infty}$. Similarly, the set of positive integers $m \geqq M$, such that $d\left(A^{m}(x), S_{2}^{+}\right)<\frac{1}{3} d\left(S_{1}^{+}, S_{2}^{+}\right)$, is also not empty. Hence, there exists a positive integer $n \geqq M$ such that both

and

$$
d\left(A^{n}(x), S_{1}^{+}\right)<\frac{1}{3} d\left(S_{1}^{+}, S_{2}^{+}\right)
$$

$$
d\left(A^{n+1}(x), S_{2}^{+}\right)<\frac{1}{3} d\left(S_{1}^{+}, S_{2}^{+}\right)
$$

(this can be seen at once, for, given a positive integer $m_{1} \geqq M$ such that $d\left(A^{m_{1}}(x), S_{1}^{+}\right)$ $<\frac{1}{3} d\left(S_{1}^{+}, S_{2}^{+}\right)$, there always exists an integer $m_{2}>m_{1}$ such that $d\left(A^{m_{2}}(x), S_{2}^{+}\right)$ $<\frac{1}{3} d\left(S_{1}^{+}, S_{2}^{+}\right)$; then $n$ can be chosen to be one less than the smallest such $\left.m_{2}\right)$. But, since

$$
d\left(S_{1}^{+}, S_{2}^{+}\right) \leqq d\left(A^{n}(x), S_{1}^{+}\right)+d\left(A^{n}(x), A^{n+1}(x)\right)+d\left(A^{n+1}(x), S_{2}^{+}\right),
$$

it would have to be true that

$$
d\left(S_{1}^{+}, S_{2}^{+}\right)<\frac{1}{3} d\left(S_{1}^{+}, S_{2}^{+}\right)+\frac{1}{3} d\left(S_{1}^{+}, S_{2}^{+}\right)+\frac{1}{3} d\left(S_{1}^{+}, S_{2}^{+}\right)=d\left(S_{1}^{+}, S_{2}^{+}\right) .
$$

Thus, the hypothesis that $\mathscr{C}(x)=S_{1} \cup S_{2}$, with $S_{1}$ and $S_{2}$ closed, nonempty, and disjoint, has been shown to lead to a contradiction.

5. $\mathscr{L}(x)$ is empty, a single point, or uncountable. Since $\mathscr{L}(x)$ is already known to be closed, connected, and compact, the desired conclusion follows from known reasoning (see, in particular, the argument in R. L. Moore [11, p. 11, Theorem 15; p. 30, Theorem 44]). However, the special situation which is of interest here requires, for completeness and clarity, a detailed proof.

It will be shown that if $\mathscr{L}(x)$ contains two or more points, then it contains uncountably many points. Suppose, contrary to what one wants to prove, that $\mathscr{L}(x)$ is countable. Since $\mathscr{L}(x)$ is connected and contains two or more points, it must contain a countable infinity of points. Let $p_{1}, p_{2}, \ldots$ denote the (distinct) points of $\mathscr{L}(x)$. Since $\mathscr{L}(x)$ is connected, each point $p_{n}$ is a limit point of the set $\mathscr{L}(x)$.

A Cauchy sequence $p_{n_{1}}, p_{n_{2}}, \ldots$, where $n_{1}<n_{2}<\cdots$, will now be extracted from $\mathscr{L}(x)$. The sequence of positive integers $n_{1}, n_{2}, \ldots$, and an auxiliary sequence of positive numbers $r_{1}, r_{2}, \ldots$, will be defined by recurrence. Let $n_{1}=1$ and $r_{1}=1$. Define $n_{2}$ to be the smallest integer $n>n_{1}$ such that $d\left(p_{n}, p_{n_{1}}\right)<r_{1}$ (since $p_{n_{1}}$ is a limit point of $\mathscr{L}(x)$, there are infinitely many such points $p_{n}$ ). Let the real number $r_{2}$ be such that

$$
0<r_{2}<\frac{1}{2} \min _{1 \leqq i<n_{2}} d\left(p_{i}, p_{n_{2}}\right)
$$


Suppose that $n_{k}$ and $r_{k}>0$ have been defined, by recurrence, for some integer $k \geqq 1$. Then $n_{k+1}$ is defined to be the smallest integer $n>n_{k}$ such that $d\left(p_{n}, p_{n_{k}}\right)<r_{k}$. Also, let the real number $r_{k+1}$ be such that

$$
0<r_{k+1}<\frac{1}{2} \min _{1 \leqq i<n_{k+1}} d\left(p_{i}, p_{n_{k+1}}\right) .
$$

In order to see that the sequence of points $p_{n_{1}}, p_{n_{2}}, \ldots$ is a Cauchy sequence, it is to be noticed first that, from the definition of $n_{k}$ and $r_{k}$, it follows that, for $k \geqq 1, d\left(p_{n_{k+1}}, p_{n_{k}}\right)<r_{k}$. Similarly, from the definitions of $n_{k+1}, r_{k+1}$, and $n_{k}$,

$$
d\left(p_{n_{k+2}}, p_{n_{k+1}}\right)<r_{k+1}<\frac{1}{2} d\left(p_{n_{k+1}}, p_{n_{k}}\right)<r_{k} / 2 .
$$

By induction, for any $i=1,2, \ldots$, one has

$$
d\left(p_{n_{k+i}}, p_{n_{k+1-1}}\right)<r_{k} / 2^{i-1} .
$$

From this, together with the triangle inequality, it now follows that

$$
\begin{aligned}
d\left(p_{n_{k+j}}, p_{n_{k}}\right) & \leqq d\left(p_{n_{k+j}}, p_{n_{k+j-1}}\right)+\cdots+d\left(p_{n_{k+1}}, p_{n_{k}}\right) \\
& <\left(\frac{1}{2^{j-1}}+\cdots+\frac{1}{2^{0}}\right) r_{k}<2 r_{k}
\end{aligned}
$$

for $k \geqq 1$ and all $j=1,2, \ldots$. Since

$$
r_{k+1}<\frac{r_{k}}{2}<\frac{r_{k-1}}{2^{2}}<\cdots<\frac{r_{1}}{2^{k}}=\frac{1}{2^{k}}
$$

it follows that $\lim _{k \rightarrow \infty} r_{k}=0$. This, together with the immediately preceding inequality, shows that the sequence of points $p_{n_{1}}, p_{n_{2}}, \ldots$ is a Cauchy sequence.

The Cauchy sequence $p_{n_{1}}, p_{n_{2}}, \ldots$ will now be used to show that $\mathscr{L}(x)$ cannot be countable. Since $\mathscr{L}(x)$ is compact, the sequence $p_{n_{1}}, p_{n_{2}}, \ldots$ contains a convergent subsequence which converges to a point $p$ of $\mathscr{L}(x)$ (because $\mathscr{L}(x)$ is closed). Since the sequence is a Cauchy sequence, the entire sequence must converge to $p$, that is $\lim _{k \rightarrow \infty} p_{n_{k}}=p$. But $p \in \mathscr{L}(x)$, therefore $p=p_{N}$ for some positive integer $N$. There exists a positive integer $k \geqq 2$ such that $N<n_{k}$. But,

$$
0<r_{k}<\frac{1}{2} \min _{1 \leqq i<n_{k}} d\left(p_{i}, p_{n_{k}}\right) \leqq \frac{1}{2} d\left(p_{N}, p_{n_{k}}\right),
$$

that is, $d\left(p_{N}, p_{n_{k}}\right)>2 r_{k}$.

On the other hand, for the same integer $k$ just considered, one has

$$
d\left(p_{n_{k+1}}, p_{n_{k}}\right)<2 r_{k}, \quad j=1,2, \ldots,
$$

as was shown before. Hence,

$$
d\left(p_{N}, p_{n_{k}}\right)=\lim _{j \rightarrow \infty} d\left(p_{n_{k+j}}, p_{n_{k}}\right) \leqq 2 r_{k},
$$

contradicting the inequality at the end of the last paragraph. Therefore, $\mathscr{S}(x)$ cannot be countable. 
6. $\mathscr{L}(x)$ consists of just one point implies that $\lim _{m \rightarrow \infty} A^{m}(x)$ exists. Since $F(A)$ is closed and compact, there exist points $p_{m} \in F(A)$ such that

$$
d\left(A^{m}(x), F(A)\right)=d\left(A^{m}(x), p_{m}\right)
$$

for $m=1,2, \ldots$ Further, since $\mathscr{L}(x)$ is nonempty, one has, by (ii) and part 2 of the proof, that the sequence $\left\{d\left(A^{m}(x), p_{m}\right)\right\}_{m=1}^{\infty}$ decreases to zero. Also, since $\mathscr{L}(x)$ consists of a single point $p \in F(A)$, there is a subsequence $\left\{A^{m_{i}}(x)\right\}_{i=1}^{\infty}$ with $\lim _{i \rightarrow \infty} A^{m_{i}}(x)=p$. It has to be shown that the whole sequence of iterates $\left\{A^{m}(x)\right\}_{m=1}^{\infty}$ converges to $p$. This will be done by first showing that the sequence $\left\{p_{m}\right\}_{m=1}^{\infty}$ converges to $p$.

Suppose that the sequence $\left\{p_{m}\right\}_{m=1}^{\infty}$ does not converge to $p$. Then there is a positive number $\varepsilon_{0}$ and a subsequence $\left\{p_{k_{1}}\right\}_{i=1}^{\infty}$ such that

$$
0<\varepsilon_{0} \leqq d\left(p_{k_{i}}, p\right), \quad i=1,2, \ldots
$$

Since $p_{k_{t}} \in F(A)$ for $i=1,2, \ldots$, and $F(A)$ is compact, the subsequence $\left\{p_{k_{i}}\right\}_{i=1}^{\infty}$ must contain a convergent subsequence (which, for convenience, will again be denoted by $\left.\left\{p_{k_{i}}\right\}_{i=1}^{\infty}\right)$. Let $q=\lim _{i \rightarrow \infty} p_{k_{\mathfrak{i}}}$, where $q \neq p$ and $q \in F(A)$. Then

where

$$
d\left(A^{k_{i}}(x), q\right) \leqq d\left(A^{k_{i}}(x), p_{k_{i}}\right)+d\left(p_{k_{i}}, q\right), \quad i=1,2, \ldots,
$$

$$
\lim _{i \rightarrow \infty} d\left(A^{k_{i}}(x), p_{k_{i}}\right)=\lim _{i \rightarrow \infty} d\left(p_{k_{i}}, q\right)=0 .
$$

Hence, $\lim _{i \rightarrow \infty} A^{k_{1}}(x)=q$, which means that also $q \in \mathscr{L}(x)$, and $\mathscr{L}(x)$ contains at least two points $p$ and $q$. This contradiction shows that $\lim _{m \rightarrow \infty} p_{m}=p$.

Now, one has that

However,

$$
d\left(A^{m}(x), p\right) \leqq d\left(A^{m}(x), p_{m}\right)+d\left(p_{m}, p\right), \quad m=1,2, \ldots
$$

$$
\lim _{m \rightarrow \infty} d\left(A^{m}(x), p_{m}\right)=\lim _{m \rightarrow \infty} d\left(p_{m}, p\right)=0,
$$

which gives $\lim _{m \rightarrow \infty} A^{m}(x)=p$.

7. $\mathscr{L}(x)$, if uncountable, lies in the boundary of $F(A)$. A point $y \in S$ is a boundary point of a set $K$ if, for every $\varepsilon>0$, there are two points, one in $K$, and one not in $K$, with both points at a distance less than $\varepsilon$ from $y$. Since $F(A)$ is closed, every boundary point of $F(A)$ belongs to $F(A)$; and a point $y$ in $F(A)$ is a boundary point of $F(A)$ if and only if, for every $\varepsilon>0$, there is a point not in $F(A)$ at a distance less than $\varepsilon$ from $y$.

Suppose $\mathscr{L}(x)$ is uncountable. Then $x \notin F(A)$ and $A^{m}(x) \notin F(A)$ for $m=1,2, \ldots$, because $\mathscr{L}(x)$ contains more than just one point. Let $y \in \mathscr{L}(x) \subseteq F(A)$. There is a subsequence of iterates $\left\{A^{m_{i}}(x)\right\}_{i=1}^{\infty}$ such that $\lim _{i \rightarrow \infty} A^{m_{i}}(x)=y$. But $A^{m_{i}}(x) \notin F(A)$ for $i=1,2, \ldots$ Therefore $y$ is a boundary point of $F(A)$.

REMARK 4. It is natural to seek to relax condition (ii) and to inquire how far the conclusion of the theorem remains unaltered. Suppose that the strict inequality in (ii) is replaced by

$$
d(A(x), F(A)) \leqq d(x, F(A)), \quad x \in S .
$$


Take $A$ to be a rotation through an angle of $180^{\circ}$ about the origin in the Euclidean plane $E_{2}$, which obviously has the origin as its only fixed point. This simple example shows that, under the modified hypothesis, it may happen that not only does $\mathscr{L}(x)$ contain points outside $F(A)$, but also that $\mathscr{L}(x)$ is not connected.

However, under an additional hypothesis, the conclusion of Theorem 2 can still be preserved, while still weakening (ii) as indicated above. This will be shown in $\$ 6$.

Corollary 2.1. Suppose that to the hypotheses of Theorem 2 one adds the assumption that, for some $x \in S$, the sequence of iterates $\left\{A^{m}(x)\right\}_{m=1}^{\infty}$ contains a convergent subsequence. Then $\mathscr{L}(x)$ is a nonempty, closed, and connected subset of $F(A)$. The rest of the conclusion of Theorem 2 remains unchanged.

Proof. The additional hypothesis, plus the definition of $\mathscr{L}(x)$, imply that $\mathscr{L}(x)$ is nonempty. This "convergent subsequence" condition appears in Edelstein [2]; and, more recently, in Browder and Petryshyn [4, part b of Theorem 1].

Corollary 2.2. Suppose, in addition to the hypotheses of Theorem 2, that $F(A)$ is a countable set. Let $x \in S$. Then, either $\left\{A^{m}(x)\right\}_{m=1}^{\infty}$ contains no convergent subsequence, or $\lim _{m \rightarrow \infty} A^{m}(x)$ exists and belongs to $F(A)$.

Proof. $\mathscr{L}(x)$ must be countable, since $\mathscr{L}(x) \subseteq F(A)$. Hence, either $\mathscr{L}(x)$ is empty or it consists of exactly one point.

COROllary 2.3. Suppose, in addition to the hypotheses of Corollary 2.2, that, for some $x \in S$, the sequence of iterates $\left\{A^{m}(x)\right\}_{m=1}^{\infty}$ contains a convergent subsequence. Then $\lim _{m \rightarrow \infty} A^{m}(x)$ exists and belongs to $F(A)$. Thus, under the above assumptions, the process of successive approximations, starting from $x$, converges to a fixed point of $A$.

In particular, Corollary 2.1, when the space $S$ is Euclidean $n$-space, yields the following generalization (to $E_{n}$ ) of Theorem 1 .

THEOREM $2\left(E_{n}\right)$. Let $A: S \rightarrow S$ be continuous, where $S$ is a nonempty closed subset of $E_{n}$. Suppose

(i) $F(A)$ is nonempty and bounded;

(ii) for each $x \in S$, with $x \notin F(A)$, one has

$$
d(A(x), F(A))<d(x, F(A)) ;
$$

where $d(x, F(A))=\inf _{p \in F(A)}\|x-p\|$, and \|\| denotes any convenient norm in $E_{n}$. Then, for $x \in S$, the set $\mathscr{L}(x)$ is a nonempty, closed, and connected subset of $F(A)$. The rest of the conclusion of Theorem 2 remains unchanged.

Proof. Since the sequence of positive numbers $\left\{d\left(A^{m}(x), F(A)\right)\right\}_{m=1}^{\infty}$ is nonincreasing, and $F(A)$ is nonempty and bounded, the set of iterates is contained in the closed and bounded set

$$
\{y \mid d(y, F(A)) \leqq d(A(x), F(A))\} .
$$

Hence, the sequence of iterates must contain a convergent subsequence. 
Remark 5. Although in $E_{n}$, the set $\mathscr{L}(x)$ is never empty (see Theorem $2\left(E_{n}\right)$ ), it may happen in some spaces that $\mathscr{L}(x)$ is empty. In seeking to construct an example, one is naturally led to consider first a real Hilbert space, with unit orthonormal base vectors $e_{1}, e_{2}, \ldots$ It seems reasonable that one will obtain a function of the desired kind by first defining its value at each $e_{n}$ to be $e_{n+1}$ (that is to say, a "shift" operator), and then extending its definition to the whole Hilbert space by linearity. The set of fixed points of this function consists of the zero vector alone. But such a function does not satisfy condition (ii); because, for example, the image of $e_{1}$, which is $e_{2}$, has the same norm as $e_{1}$. However, a slight modification leads to the following example.

Let $\alpha_{1}, \alpha_{2}, \ldots$ be a strictly increasing sequence of positive numbers such that

$$
\lim _{i \rightarrow \infty} \alpha_{i}=1 \text { and } \prod_{i=1}^{\infty} \alpha_{i}=\frac{1}{\pi} .
$$

In particular, one might choose

$$
\alpha_{1}=\frac{1}{2}, \quad \alpha_{2}=1-\frac{1}{4 \cdot 1^{2}}, \quad \alpha_{3}=1-\frac{1}{4 \cdot 2^{2}}, \quad \alpha_{4}=1-\frac{1}{4 \cdot 3^{2}}, \ldots,
$$

which is obtained by putting $\theta=\pi / 2$ in Euler's infinite product for the sine function:

$$
\sin \theta=\theta\left(1-\frac{\theta^{2}}{\pi^{2}}\right)\left(1-\frac{\theta^{2}}{2^{2} \pi^{2}}\right)\left(1-\frac{\theta^{2}}{3^{2} \pi^{2}}\right) \cdots
$$

Let the function $A$ be defined first on the unit vectors, by putting $A e_{n}=\alpha_{n} e_{n+1}$, $n=1,2, \ldots$; and then extend its definition to the whole Hilbert space by linearity. Then, for every vector $x=\sum_{i=1}^{\infty} x_{i} e_{i}$ in the Hilbert space, one has

Further, if $y=\sum_{i=1}^{\infty} y_{i} e_{i}$, then

$$
A(x)=\sum_{i=1}^{\infty} \alpha_{i} x_{i} e_{i+1}
$$

$$
\|A(x)-A(y)\|^{2}=\sum_{i=1}^{\infty} \alpha_{i}^{2}\left(x_{i}-y_{i}\right)^{2} \leqq \sum_{i=1}^{\infty}\left(x_{i}-y_{i}\right)^{2}=\|x-y\|^{2},
$$

where equality holds if and only if $x=y$. Consequently, $A$ is (strongly) continuous. Putting $y=0$, one obtains that $\|A(x)\| \leqq\|x\|$, with equality if and only if $x=0$, so that $F(A)$ consists of the zero vector alone, and hypotheses (i) and (ii) are satisfied. In view of Theorem 2 , for any $x \neq 0$, the set $\mathscr{L}(x)$ is either empty or is just the zero vector. Let $x \neq 0$, then, by induction,

so that

$$
A^{m}(x)=\sum_{i=1}^{\infty} \alpha_{i} \alpha_{i+1} \cdots \alpha_{i+m-1} x_{i} e_{i+m}, \quad m=1,2, \ldots,
$$

$$
\begin{aligned}
\left\|A^{m}(x)\right\|^{2} & =\sum_{i=1}^{\infty}\left(\alpha_{i} \alpha_{i+1} \cdots \alpha_{i+m-1}\right)^{2} x_{i}^{2} \\
& \geqq \sum_{i=1}^{\infty}\left(\prod_{j=1}^{\infty} \alpha_{j}\right)^{2} x_{i}^{2}=\frac{1}{\pi^{2}} \sum_{i=1}^{\infty} x_{i}^{2} .
\end{aligned}
$$


Therefore, the sequence $\left\{A^{m}(x)\right\}_{m=1}^{\infty}$ cannot contain any subsequence which converges to the zero vector, and $\mathscr{L}(x)$ must be empty.

ReMARK 6. In one dimension, the conclusion of Theorem $2\left(E_{n}\right)$ can be restated in an equivalent, seemingly stronger form:

THEOREM $2\left(E_{1}\right)$. Let $A: S \rightarrow S$ be continuous, where $S$ is a nonempty closed subset of $E_{1}$. Suppose

(i) $F(A)$ is nonempty and bounded;

(ii) for each $x \in S$, with $x \notin F(A)$, one has

$$
d(A(x), F(A))<d(x, F(A)) .
$$

Then, for $x \in S, \lim _{m \rightarrow \infty} A^{m}(x)$ exists and belongs to $F(A)$.

Proof. Putting $n=1$ in Theorem $2\left(E_{n}\right)$, one deduces that $\mathscr{L}(x)$ is a nonempty, closed, and connected subset of $F(A) \subseteq E_{1} . \mathscr{L}(x)$ is either a nondegenerate closed interval contained in the boundary of $F(A)$ or a single number in $F(A)$. But the boundary of any set in $E_{1}$ can never contain a nondegenerate closed interval. Therefore, $\mathscr{L}(x)$ must reduce to a single number.

The present theorem is a generalization of the "modified Tricomi result" of Remark 3 of $\S 2$, since here the set $F(A)$ is not restricted to consist of a single number. (However, this is only a slight generalization; because, if (ii) is to hold, then $F(A)$ can be shown to consist of a single closed interval.)

In [12, Theorem 1], Chu and Moyer have shown, among other results, that if $A$ is a continuous mapping of the finite closed interval $[a, b]$ into itself, then the following two conditions are equivalent:

(j) for each $x \in[a, b]$, such that $x \notin F(A)$, one has $A^{2}(x) \neq x$;

(ji) the sequence of iterates $\left\{A^{m}(x)\right\}_{m=1}^{\infty}$ converges for each $x \in[a, b]$.

It is of interest to notice that, when $S=[a, b]$ in Theorem $2\left(E_{1}\right)$, hypothesis (i) of that theorem is automatically fulfilled; hypothesis (ii) implies condition (j); and the conclusion of Theorem $2\left(E_{1}\right)$ is just condition (jj). That hypothesis (ii) implies condition (j) may be seen as follows. If $x \notin F(A)$ and $A(x) \in F(A)$, then $A^{2}(x)=A(x) \neq x$, which is (j). On the other hand, if $x \notin F(A)$ and $A(x) \notin F(A)$, then

$$
d\left(A^{2}(x), F(A)\right)<d(A(x), F(A))<d(x, F(A)),
$$

which gives $A^{2}(x) \neq x$, as desired.

REMARK 7. It is of interest to observe that when $S$ is a closed convex set in $E_{n}$, and the function $A$ is a continuously differentiable function satisfying a "norm condition" of the form

$$
\left\|\left(\partial A_{j} / \partial x_{i}\right)\right\|<1,
$$

one can deduce from Theorem $2\left(E_{n}\right)$ a result concerning "points of attraction for iterations with several variables" (for details and bibliography see Ostrowski $[8$, p. 119, Theorem 18.1]). 
The following Theorem $2^{k}$ is concerned with an iterate $A^{k}$, rather than with $A$ itself. Thus, Theorem $2^{k}$ generalizes Theorem 2 , in the same way in which Theorem 1.1 generalizes Theorem 1 .

Theorem $2^{k}$. Let $A: S \rightarrow S$, where $S$ is a nonempty metric space, and $A^{k}$ is continuous for some positive integer $k$. Suppose

(i) $F\left(A^{k}\right)$ is nonempty and compact;

(ii) for each $x \in S$, with $x \notin F(A)$, one has

$$
d\left(A^{k}(x), F\left(A^{k}\right)\right)<d\left(x, F\left(A^{k}\right)\right) .
$$

Then, for $x \in S$, the set $\mathscr{L}_{k}(x)$ of subsequential limit points of the sequence of iterates $\left\{A^{m k}(x)\right\}_{m=1}^{\infty}$ is a closed and connected subset of $F\left(A^{k}\right)$. Further, for $x \in S$, the set $\mathscr{L}_{1}(x)$ of subsequential limit points of the sequence of iterates $\left\{A^{m}(x)\right\}_{m=1}^{\infty}$ is the union of the $k$ closed and connected sets $\mathscr{L}_{k}\left(A^{j}(x)\right)$, where $j=0,1, \ldots, k-1$. That is,

$$
\mathscr{L}_{1}(x)=\bigcup_{j=0}^{k-1} \mathscr{L}_{k}\left(A^{j}(x)\right)
$$

Proof. From Theorem 2, applied to the function $A^{k}$, it follows that $\mathscr{L}_{k}(x)$ is a closed and connected subset of $F\left(A^{k}\right)$. Therefore, it only remains to prove the "formula" for $\mathscr{L}_{1}(x)$.

First it will be shown that

$$
\mathscr{L}_{1}(x) \supseteq \bigcup_{j=0}^{k-1} \mathscr{L}_{k}\left(A^{j}(x)\right)
$$

If $\mathscr{L}_{k}\left(A^{j}(x)\right)$ is empty for every $j=0,1, \ldots, k-1$, then there is nothing to prove. Therefore, suppose that, for some $0 \leqq j \leqq k-1, \mathscr{L}_{k}\left(A^{j}(x)\right)$ is nonempty, and let $z \in \mathscr{L}_{k}\left(A^{j}(x)\right)$. Then there exists a sequence of iterates $\left\{A^{m_{i} k+j}(x)\right\}_{i=1}^{\infty}$ which converges to $z$. Hence, $z \in \mathscr{L}_{1}(x)$. That is, for each $j=0,1, \ldots, k-1$ for which $\mathscr{L}_{k}\left(A^{j}(x)\right)$ is nonempty one has $\mathscr{L}_{i}\left(A^{j}(x)\right) \subseteq \mathscr{L}_{1}(x)$.

Next it will be shown that

$$
\mathscr{L}_{1}(x) \subseteq \bigcup_{j=0}^{k-1} \mathscr{L}_{k}\left(A^{j}(x)\right)
$$

If $\mathscr{L}_{1}(x)$ is empty, then there is nothing to prove. Therefore, suppose that $\mathscr{L}_{1}(x)$ is nonempty, and let $z \in \mathscr{L}_{1}(x)$. Then there exists a sequence of iterates $\left\{A^{m_{i}}(x)\right\}_{i=1}^{\infty}$ which converges to $z$. There is an infinite subsequence of $\left\{m_{i}\right\}_{i=0}^{\infty}$ which is contained in one of the $k$ infinite sequences

$$
\{m k+j\}_{m=0}^{\infty}, \quad j=0,1, \ldots, k-1 .
$$

Denote this subsequence by $\left\{m_{i}^{\prime} k+j\right\}_{i=0}^{\infty}$, where $j=j(z)$. Then

$$
\lim _{i \rightarrow \infty} A^{m_{i}^{\prime} k+j}(x)=\lim _{i \rightarrow \infty} A^{m_{i}^{\prime} k}\left(A^{j}(x)\right)=z ;
$$

hence $z \in \mathscr{L}_{k}\left(A^{j}(x)\right)$, and the proof is complete. 
Corollary $2^{k} .1$. Suppose, in addition to the hypotheses of Theorem $2^{k}$, that $F\left(A^{k}\right)$ is a countable set. Let $x \in S$. Then $\mathscr{L}_{1}(x)$ contains at most $k$ points.

Proof. Each set $\mathscr{L}_{k}\left(A^{j}(x)\right), j=0,1, \ldots, k-1$, is a closed and connected subset of $F\left(A^{k}\right)$. Therefore, each $\mathscr{L}_{k}\left(A^{j}(x)\right)$ must be empty or consist of one point. But, by Theorem $2^{k}$,

which gives the result.

$$
\mathscr{L}_{1}(x)=\bigcup_{j=0}^{k-1} \mathscr{L}_{k}\left(A^{j}(x)\right)
$$

REMARK 8. The simple example of Remark 3 serves to illustrate Theorem $2^{k}$ and Corollary $2^{k}$.1. In this example, $k=2$, the set $F\left(A^{2}\right)$ is the interval $-1 \leqq x \leqq+1$, and the set $\mathscr{L}_{1}(x)$, for $1<|x|<2$, consists of the two numbers -1 and +1 . Thus, the set $\mathscr{L}_{1}(x)$ is not connected, but is the union of the two connected sets $\mathscr{L}_{2}(x)$ $=\{-1\}$ and $\mathscr{L}_{2}(A(x))=\{+1\}$.

4. Variations. The following theorem is a variation of Theorem 2, in that hypothesis (i) of Theorem 2 is weakened, hypothesis (ii) of Theorem 2 is strengthened, and the conclusion of Theorem 2 is strengthened. Specifically, in hypothesis (i), the assumption that the set of fixed points $F(A)$ is compact will be removed; while hypothesis (ii) will be replaced by a "uniform" condition, $\left(\mathrm{ii}_{\mathrm{u}}\right)$, uniform with respect to the fixed point set $F(A)$; and the conclusion of Theorem 2 is strengthened to the assertion that $\mathscr{L}(x)$ is either empty or consists of exactly one point.

Theorem 3. Let $A: S \rightarrow S$ be continuous. Suppose

(i) $F(A)$ is nonempty;

(ii $\left.{ }_{u}\right)$ for each $x \in S$, with $x \notin F(A)$, and each $p \in F(A)$, one has $d(A(x), p)<d(x, p)$.

Let $x \in S$. Then, either $\left\{A^{m}(x)\right\}_{m=1}^{\infty}$ contains no convergent subsequence, or $\lim _{m \rightarrow \infty} A^{m}(x)$ exists and belongs to $F(A)$.

Proof. 1. If $\mathscr{L}(x)$ is empty, then there is nothing left to prove. Therefore, throughout the rest of the argument it will be supposed that $\mathscr{L}(x)$ is nonempty. If $x \in F(A)$, or if $A^{k}(x) \in F(A)$ for some integer $k \geqq 1$, then $\lim _{m \rightarrow \infty} A^{m}(x)$ exists and belongs to $F(A)$. Therefore, it will also be supposed throughout the remainder of the argument that $A^{m}(x) \notin F(A)$ for every $m=0,1,2, \ldots$ Then, for any $p \in F(A)$, the sequence of positive numbers $\left\{d\left(A^{m}(x), p\right)\right\}_{m=1}^{\infty}$ is a decreasing sequence, because, from $\left(\mathrm{ii}_{\mathrm{u}}\right)$,

$$
d\left(A^{m+1}(x), p\right)=d\left(A\left(A^{m}(x)\right), p\right)<d\left(A^{m}(x), p\right)
$$

for $m=1,2, \ldots$; hence, $\lim _{m \rightarrow \infty} d\left(A^{m}(x), p\right)$ exists and is nonnegative.

2. $\mathscr{L}(x)$ is a subset of $F(A)$. Let $\left\{A^{m_{i}}(x)\right\}_{i=1}^{\infty}$ be a convergent subsequence of $\left\{A^{m}(x)\right\}_{m=1}^{\infty}$. If it were true that $\lim _{i \rightarrow \infty} A^{m_{i}}(x) \notin F(A)$, then one would have that, for $p \in F(A)$, by the continuity of the distance function $d$, the continuity of $A$, and $\left(\mathrm{ii}_{\mathrm{u}}\right)$,

$$
\begin{aligned}
\lim _{i \rightarrow \infty} d\left(A^{m_{i}+1}(x), p\right) & =d\left(\lim _{i \rightarrow \infty} A\left(A^{m_{i}}(x)\right), p\right)=d\left(A\left(\lim _{i \rightarrow \infty} A^{m_{i}}(x)\right), p\right) \\
& <d\left(\lim _{i \rightarrow \infty} A^{m_{i}}(x), p\right)=\lim _{i \rightarrow \infty} d\left(A^{m_{i}}(x), p\right),
\end{aligned}
$$


which is a contradiction, since $\lim _{m \rightarrow \infty} d\left(A^{m}(x), p\right)$ exists. Consequently,

and hence $\mathscr{L}(x) \subseteq F(A)$.

$$
\lim _{i \rightarrow \infty} A^{m_{i}}(x) \in F(A)
$$

3. $\mathscr{L}(x)$ contains at most one point. Suppose, contrary to what one wants to prove, that $\mathscr{L}(x)$ contains at least two distinct points $p$ and $q$. Then there are strictly increasing sequences of positive integers, $\left\{m_{i}\right\}_{i=1}^{\infty}$ and $\left\{n_{i}\right\}_{i=1}^{\infty}$, such that

$$
\lim _{i \rightarrow \infty} A^{m_{i}}(x)=p \quad \text { and } \quad \lim _{i \rightarrow \infty} A^{n_{i}}(x)=q .
$$

There is a subsequence $\left\{m_{i}^{\prime}\right\}_{i=1}^{\infty}$ of $\left\{m_{i}\right\}_{i=1}^{\infty}$ such that $m_{i}^{\prime}>n_{i}$ for $i=1,2, \ldots$ Then $\lim _{i \rightarrow \infty} A^{m_{i}^{\prime}}(x)=p$.

Further, since $p \neq q$, one must have $A^{m}(x) \notin F(A)$ for $m=1,2, \ldots$ But now condition ( $\mathrm{ii}_{\mathrm{u}}$ ) gives (since $q \in F(A)$ )

$$
d\left(A^{m_{i}^{\prime}}(x), q\right)<d\left(A^{m_{i}^{\prime}-1}(x), q\right)<\cdots<d\left(A^{n_{i}}(x), q\right)
$$

which, since $\lim _{i \rightarrow \infty} d\left(A^{n_{i}}(x), q\right)=0$, implies that $\lim _{i \rightarrow \infty} A^{m_{i}^{i}}(x)=q$, contradicting $p \neq q$. Consequently, $\mathscr{L}(x)$ contains at most one point.

4. $\mathscr{L}(x)$ consists of just one point implies that $\lim _{m \rightarrow \infty} A^{m}(x)$ exists. Let the subsequence $\left\{A^{m_{i}}(x)\right\}_{i=1}^{\infty}$ be such that $\lim _{i \rightarrow \infty} A^{m_{i}}(x)=p$. Thus, for every $\varepsilon>0$, there exists a positive integer $I=I(\varepsilon)$ such that $d\left(A^{m_{i}}(x), p\right)<\varepsilon$ for all $i \geqq I$. Then, whenever $m>m_{I}$, one has

$$
d\left(A^{m}(x), p\right)<d\left(A^{m-1}(x), p\right)<\cdots<d\left(A^{m_{I}}(x), p\right)<\varepsilon,
$$

which means that $\lim _{m \rightarrow \infty} A^{m}(x)=p$.

Remark 9. Consider again the example given in Remark 5, following Theorem $2\left(E_{n}\right)$. This example shows that the possibility that $\mathscr{L}(x)$ is empty cannot be excluded from the conclusion of Theorem 3.

COROLlary 3.1. Suppose, in addition to the hypotheses of Theorem 3, that, for some $x \in S$, the sequence of iterates $\left\{A^{m}(x)\right\}_{m=1}^{\infty}$ contains a convergent subsequence. Then $\lim _{m \rightarrow \infty} A^{m}(x)$ exists and belongs to $F(A)$. Thus, under the above assumptions, the process of successive approximations, starting from $x$, converges to a fixed point of $A$.

REMARK 10. It is of interest to notice a connection with the work of Edelstein [2, Theorem 1 and 3.2]. Edelstein assumes, for every $x, y \in S$, with $x \neq y$,

$$
d(A(x), A(y))<d(x, y),
$$

and that, for some $x \in S$, there exists a convergent subsequence of iterates $\left\{A^{m_{i}}(x)\right\}_{i=1}^{\infty}$. He then concludes that $\lim _{m \rightarrow \infty} A^{m}(x)$ exists and is a fixed point of $A$. In brief, in the present terminology, Edelstein assumes that $\mathscr{L}(x) \neq \varnothing$, and that " $A$ is a contraction with respect to $S$ ", and he deduces from this that $\mathscr{L}(x) \subseteq F(A)$ 
and that $\mathscr{L}(x)$ consists of a single element. On the other hand, the present Theorem 3 assumes that $F(A) \neq \varnothing$ and that " $A$ is a contraction with respect to $F(A)$ ", and concludes that $\mathscr{L}(x) \subseteq F(A)$ and that $\mathscr{L}(x)$ is either empty or consists of a single point.

5. Applications. Corollary 3.1 may be used to prove the following theorem, which for $\lambda=\frac{1}{2}$, reduces to a theorem of Edelstein [3]; which, in turn, is an improvement of an earlier theorem of Krasnosel'skiǐ [9]. In Edelstein's own notation, the theorem in question may be formulated thus:

THEOREM 4. Let $K$ be a closed convex subset of a strictly convex Banach space $X ; f: K \rightarrow K$ a continuous mapping satisfying

$$
\|f(x)-f(y)\| \leqq\|x-y\| \quad(x, y \in K) ;
$$

and suppose $f(K)$ is contained in a compact subset $K_{1}$ of $K$. Then, for $x \in K$, the sequence $\left\{A^{m}(x)\right\}_{m=1}^{\infty}$, where $A: K \rightarrow K$ is the mapping defined by

$$
A(y)=\lambda f(y)+(1-\lambda) y \quad(y \in K), \quad 0<\lambda<1,
$$

converges to a fixed point of $f$.

Proof. Take $S=K$ in Corollary 3.1. The set of fixed points of $A$ coincides with the set of fixed points of $f$. Since $K$ is closed and convex, and $f(K)$ is compact, it follows by Schauder's theorem that the set of fixed points of $f$ is nonempty. Hence, condition (i) of Theorem 3 holds.

Let $p$ be a fixed point of $f$, i.e., $f(p)=p$. Then, from the assumed Lipschitz condition satisfied by $f$, one has $\|f(x)-p\| \leqq\|x-p\|$; hence, using the definition of $A$,

$$
\|A(x)-p-(1-\lambda)(x-p)\|=\|\lambda f(x)-\lambda p\| \leqq \lambda\|x-p\| .
$$

However, by the triangle inequality,

$$
\|A(x)-p-(1-\lambda)(x-p)\| \geqq\|A(x)-p\|-(1-\lambda)\|x-p\| .
$$

Taken together, the last two inequalities yield $\|A(x)-p\| \leqq\|x-p\|$. If $x$ is not a fixed point of $f$, then $x \neq p$, and the open line segment joining the points $x$ and $f(x)$ must, by strict convexity, be contained in the open sphere of radius $\|x-p\|$ and centered at $p$. Since $A(x)$ is an interior point of this line segment, one has $\|A(x)-p\|<\|x-p\|$. Hence, condition (ii ${ }_{\mathrm{u}}$ ) of Theorem 3 also holds.

Let $x \in K$. The closed convex hull of the set $K_{1} \cup\{x\}$ is compact, by a theorem of Mazur [13]. Since the sequence of iterates $\left\{A^{m}(x)\right\}_{m=1}^{\infty}$ is contained in this closed convex hull, this sequence must contain a convergent subsequence. Thus, all the hypotheses of Corollary 3.1 are fulfilled.

The following application of Theorem 3 seems to be of interest. It should also be remarked that the inequality appearing in condition (ii) below is reminiscent of the inequality

$$
0<\operatorname{Re}(T x-T y, x-y), \quad x, y \in H,
$$


which defines the "monotonicity" of a function $T$ on a Hilbert space $H$ to itself; see Browder [14] and Minty [15].

TheOREM 5. Let $T: H \rightarrow H$ be continuous, where $H$ is a nonempty Hilbert space. Let $y \in H$. Suppose

(i) there exists a $p \in H$ such that $T p=y$;

(ii) there exists a number $\lambda>0$ such that, for each $x \in H$, with $T x \neq y$, and each $p \in H$, with $T p=y$, one has

$$
\frac{1}{2} \lambda\|T x-T p\|^{2}<\operatorname{Re}(T x-T p, x-p) .
$$

Let $x_{0} \in H$. Then, the sequence $\left\{x_{m}\right\}_{m=0}^{\infty}$, where

$$
x_{m+1}=x_{m}+\lambda\left(y-T x_{m}\right), \quad m=0,1, \ldots,
$$

either contains no (strongly) convergent subsequence, or else $\left\{x_{m}\right\}_{m=0}^{\infty}$ is (strongly) convergent and

$$
T\left(\lim _{m \rightarrow \infty} x_{m}\right)=y .
$$

Proof. Define the function $A$ by $A(x)=x+\lambda(y-T x)$ for each $x \in H$. The set of fixed points of $A$, that is $F(A)$, corresponds to the set of $p \in H$ such that $T p=y$; hence, hypothesis (i) means that $F(A)$ is nonempty.

If $F(A)=H$, then there is nothing to prove. Suppose, therefore, that there is an $x \in H$ with $x \notin F(A)$. Let $p \in F(A)$. Then, since $A(x)=x+\lambda(T p-T x)$, one has

$$
\begin{aligned}
\|A(x)-p\|^{2} & =\|x-p\|^{2}+\lambda^{2}\|T p-T x\|^{2}+2 \lambda \operatorname{Re}(T p-T x, x-p) \\
& =\|x-p\|^{2}+\lambda\left[\lambda\|T x-T p\|^{2}-2 \operatorname{Re}(T x-T p, x-p)\right] \\
& <\|x-p\|^{2}
\end{aligned}
$$

using hypothesis (ii).

Thus, with $S=H$, and $d(u, v)=\|u-v\|$, the function $A$ satisfies the hypotheses (i) and $\left(\mathrm{ii}_{\mathrm{u}}\right)$ of Theorem 3. Consequently, for $x_{0} \in H$, the sequence $\left\{A^{m}\left(x_{0}\right)\right\}_{m=1}^{\infty}$ either contains no convergent subsequence, or $\lim _{m \rightarrow \infty} A^{m}\left(x_{0}\right)$ exists and belongs to $F(A)$, which is just a rewording of the conclusion of Theorem 5 .

Two examples will now be given, illustrating the two possible alternatives occurring in the conclusion of Theorem 5.

EXAMPLE 1. This example shows that the sequence $\left\{x_{m}\right\}_{m=0}^{\infty}$ may contain no convergent subsequence. Let the function $A$ be as in the example immediately following Theorem $2\left(E_{n}\right)$. Define $T$ by $T x=x-A(x)$ for $x \in H$. Since $A(x)=0$ only for $x=0$, it follows that $T x=0$ only for $x=0$. Recall that $\|A(x)\|<\|x\|$ for $x \neq 0$. Then one has

$$
\|A x\|^{2}=\|x-T x\|^{2}=\|x\|^{2}+\|T x\|^{2}-2 \operatorname{Re}(x, T x)<\|x\|^{2}
$$

for $x \neq 0$; thus, $\|T x\|^{2}<2 \operatorname{Re}(x, T x)$ for $x \neq 0$. Let $y=0$. Then hypothesis (i) of Theorem 5 holds with $p=0$, and the last inequality shows that hypothesis (ii) 
holds with $\lambda=1$. The sequence of iterates $\left\{A^{m}\left(x_{0}\right)\right\}_{m=1}^{\infty}$ contains no convergent subsequence when $x_{0} \neq 0$, as was shown in the earlier example. But, by the definition of $T$, using $\lambda=1$ and $p=0$ in the definition of $x_{m+1}$ in Theorem 5, one has $x_{m+1}$ $=x_{m}-T x_{m}=A\left(x_{m}\right)$ for $m=0,1,2, \ldots$; and hence, $\left\{x_{m}\right\}_{m=1}^{\infty}=\left\{A^{m}\left(x_{0}\right)\right\}_{m=1}^{\infty}$.

EXAMPLE 2. This example shows that the sequence $\left\{x_{m}\right\}_{m=0}^{\infty}$ may actually converge (strongly), and that

$$
T\left(\lim _{m \rightarrow \infty} x_{m}\right)=y .
$$

Suppose $T \not \equiv 0$ is a linear (additive and homogeneous), completely continuous (the image of every bounded sequence contains a strongly convergent subsequence), positive semidefinite (i.e., $(T x, x) \geqq 0$ for each $x \in H$ ), selfadjoint operator on $H$ to itself. Let $y$ be an element of the range of $T$. Then hypothesis (i) holds for some $p \in H$. Let $\lambda_{i}$ and $\varphi_{i}, i=1,2, \ldots$, (where $T \varphi_{i}=\lambda_{i} \varphi_{i}, \lambda_{1} \geqq \lambda_{2} \geqq \cdots$, and $\lambda_{1}>0$ ), be the eigenvalues and eigenvectors, respectively, of $T$. Then, for any $z \in H$,

$$
z=z^{\perp}+\sum_{i=1}^{\infty} z_{i} \varphi_{i}
$$

where the vector $z^{\perp}$ is orthogonal to all the eigenvectors (i.e., $\left(z^{\perp}, \varphi_{i}\right)=0$ for $i=$ $1,2, \ldots)$ and $z_{i}=\left(z, \varphi_{i}\right)$ for $i=1,2, \ldots$ Also,

and

$$
T z=\sum_{i=1}^{\infty} \lambda_{i} z_{i} \varphi_{i}
$$

$$
(T z, z)-\frac{\lambda}{2}\|T z\|^{2}=\sum_{i=1}^{\infty} \lambda_{i} z_{i}^{2}-\frac{\lambda}{2} \sum_{i=1}^{\infty} \lambda_{i}^{2} z_{i}^{2}=\sum_{i=1}^{\infty}\left(1-\frac{\lambda}{2} \cdot \lambda_{i}\right) \lambda_{i} z_{i}^{2} .
$$

Suppose that $\lambda$ satisfies $0<\lambda<2 / \lambda_{1}$; then $1-\lambda / 2 \cdot \lambda_{i} \geqq 1-\lambda / 2 \cdot \lambda_{1}>0$ for $i=1,2, \ldots$ Making use of this, and putting $z=x-p$, where $T p=y$ and $T x \neq y$, one obtains

$$
(T(x-p), x-p)-\frac{\lambda}{2}\|T(x-p)\|^{2}>0
$$

so that hypothesis (ii) holds.

When applied in this particular instance, the conclusion of Theorem 5 only gives an alternative, that is, either $\left\{x_{m}\right\}_{m=0}^{\infty}$ converges or else it contains no convegent subsequence. However, it will now be shown that, actually, the sequence $\left\{x_{m}\right\}_{m=0}^{\infty}$ converges (strongly). Suppose that $T p=y$, and let

$$
x_{0}=x_{0}^{\perp}+\sum_{i=1}^{\infty} x_{0 i} \varphi_{i},
$$

where $x_{0}^{\frac{1}{1}}$ is orthogonal to all the eigenvectors, and $x_{0 i}=\left(x_{0}, \varphi_{i}\right)$ for $i=1,2, \ldots$. Using the definition, by recurrence, of $x_{m}$, it may be readily shown by mathematical induction that

$$
x_{m}=p+x_{0}^{\perp}+\sum_{i=1}^{\infty}\left(1-\lambda \lambda_{i}\right)^{m}\left(x_{0 i}-p_{i}\right) \varphi_{i}
$$


for $m \geqq 1$, where $p_{i}=\left(p, \varphi_{i}\right)$ for $i=1,2, \ldots$ It now only remains to prove that, as $m \rightarrow \infty$, the vector

$$
\sum_{i=1}^{\infty}\left(1-\lambda \lambda_{i}\right)^{m}\left(x_{0 i}-p_{i}\right) \varphi_{i}
$$

converges (strongly) to zero; since, if this is the case, $\left\{x_{m}\right\}_{m=0}^{\infty}$ converges (strongly) to $p+x_{0}^{1}$ and

$$
T\left(\lim _{m \rightarrow \infty} x_{m}\right)=T\left(p+x_{0}^{\perp}\right)=T p=y .
$$

The asserted strong convergence follows from the equation

$$
\begin{aligned}
\left\|\sum_{i=1}^{\infty}\left(1-\lambda \lambda_{i}\right)^{m}\left(x_{0 i}-p_{i}\right) \varphi_{i}\right\|^{2}= & \sum_{i=1}^{k}\left(1-\lambda \lambda_{i}\right)^{2 m}\left(x_{0 i}-p_{i}\right)^{2} \\
& +\sum_{i=k+1}^{\infty}\left(1-\lambda \lambda_{i}\right)^{2 m}\left(x_{0 i}-p_{i}\right)^{2},
\end{aligned}
$$

which is valid for any $k=1,2, \ldots$ Let $\varepsilon>0$. Choose $k$ so large that (using $\left.-1<1-\lambda \lambda_{i}<+1\right)$

$$
\sum_{i=k+1}^{\infty}\left(1-\lambda \lambda_{i}\right)^{2 m}\left(x_{0 i}-p_{i}\right)^{2} \leqq \sum_{i=k+1}^{\infty}\left(x_{0 i}-p_{i}\right)^{2}<\frac{\varepsilon}{2}
$$

Having chosen $k$, it is true that, for all sufficiently large $m$, one has

$$
\sum_{i=1}^{k}\left(1-\lambda \lambda_{i}\right)^{2 m}\left(x_{0 i}-p_{i}\right)^{2}<\frac{\varepsilon}{2}
$$

Thus, for all sufficiently large $m$,

$$
\left\|\sum_{i=1}^{\infty}\left(1-\lambda \lambda_{i}\right)^{m}\left(x_{0 i}-p_{i}\right) \varphi_{i}\right\|^{2}<\varepsilon,
$$

and the proof is complete.

REMARK 11. Fridman [10], in the case of a Fredholm integral equation of the first kind with a positive definite continuous kernel, has given the convergence theorem which is implicitly contained in Example 2 of Remark 10.

ReMARK 12. Hypothesis (i) of Theorem 5 just states that $y$ belongs to the range of $T$. This hypothesis, for a different class of operators, is shown in Browder and Petryshyn [4, Theorem 1, part a] to imply the convergence of successive approximations.

6. Some further modifications, involving "asymptotic regularity". Theorems 2.1 and 3.1 of this section are variations of Theorems 2 and 3, involving the additional hypothesis (iii) (see below). This hypothesis (iii), in a Banach space, was termed "asymptotic regularity" by Browder and Petryshyn [5, p. 572, Definition 1]. With the addition of hypothesis (iii), it becomes possible to replace (ii) of Theorem 2 by the weaker hypothesis $\left(\mathrm{ii}_{\mathrm{w}}\right)$ (see below). 
THEOREM 2.1. Let $A: S \rightarrow S$ be continuous, where $S$ is a nonempty metric space. Suppose

(i) $F(A)$ is nonempty and compact;

(ii $\left.{ }_{\mathrm{w}}\right)$ for each $x \in S$, one has

$$
d(A(x), F(A)) \leqq d(x, F(A)) ;
$$

(iii) for each $x \in S$, one has

$$
\lim _{m \rightarrow \infty} d\left(A^{m+1}(x), A^{m}(x)\right)=0 .
$$

Then, for $x \in S$, the set $\mathscr{L}(x)$ is a closed and connected subset of $F(A)$. Either $\mathscr{L}(x)$ is empty, or it contains exactly one point, or it contains uncountably many points. In case $\mathscr{L}(x)$ is just one point, then $\lim _{m \rightarrow \infty} A^{m}(x)$ exists and belongs to $F(A)$. In case $\mathscr{L}(x)$ is uncountable, then it is contained in the boundary of $F(A)$.

Proof. The essential differences between this proof and the proof of Theorem 2 are:

(1) From (ii $\mathrm{w}_{\mathrm{w}}$ it follows that the sequence of nonnegative numbers $\left\{d\left(A^{m}(x), F(A)\right)\right\}_{m=1}^{\infty}$ is nonincreasing for any $x \in S$ (recall that, in the proof of Theorem 2 , this sequence is strictly decreasing whenever $x$ is such that $A^{m}(x) \notin F(A)$ for $m=0,1,2, \ldots)$;

(2) Part 2 of the proof of Theorem 2 is replaced with a proof that $\mathscr{L}(x) \subseteq F(A)$, based only on hypothesis (iii) and the continuity of $A$, and nothing else.

In the proof of Theorem 2, essential use of the knowledge that $\left\{d\left(A^{m}(x), F(A)\right)\right\}_{m=1}^{\infty}$ is decreasing, is made only in part 2. But part 2 will now be replaced by the following argument. If $\mathscr{L}(x)=\varnothing$, then $\mathscr{L}(x) \subseteq F(A)$. If $\mathscr{L}(x) \neq \varnothing$, suppose $z \in \mathscr{L}(x)$ and that $\lim _{i \rightarrow \infty} A^{m_{i}}(x)=z$. Then, by the continuity of $A$,

$$
\lim _{i \rightarrow \infty} A^{m_{i}+1}(x)=A\left(\lim _{i \rightarrow \infty} A^{m_{i}}(x)\right)=A(z) .
$$

Hence, from (iii),

$$
\begin{aligned}
d(A(z), z) & =d\left(\lim _{i \rightarrow \infty} A^{m_{i}+1}(x), \lim _{i \rightarrow \infty} A^{m_{i}}(x)\right) \\
& =\lim _{i \rightarrow \infty} d\left(A^{m_{i}+1}(x), A^{m_{i}}(x)\right)=0,
\end{aligned}
$$

so that $A(z)=z$, and thus $z \in F(A)$.

In several places in the proof of Theorem 2, other than part 2, use is made of the fact that $\left\{d\left(A^{m}(x), F(A)\right)\right\}_{m=1}^{\infty}$ is decreasing to zero. In all these places one can now use the fact that $\left\{d\left(A^{m}(x), F(A)\right)\right\}_{m=1}^{\infty}$ is nonincreasing and tends to zero (provided $\mathscr{L}(x)$ is nonempty).

It is to be noticed that, in the proof of Theorem 2, part 4, the preliminary argument amounts to showing that (iii) holds, under both hypothesis (ii) and the assumption that $\mathscr{L}(x) \neq \varnothing$. Hence, in the present theorem, this preliminary argument is avoided, simply by assuming (iii). However, if hypothesis (ii) holds, but $\mathscr{L}(x)=\varnothing$, 
it may happen that (iii) does not hold, as may be seen from the example of Remark 5 following Theorem 2 . Hence, hypothesis (ii), by itself, does not imply hypothesis (iii).

Theorem 3.1 below is obtained from Theorem 3 upon adding hypothesis (iii), while replacing $\left(\mathrm{ii}_{\mathrm{u}}\right)$ by the weaker hypothesis $\left(\mathrm{ii}_{\mathrm{uw}}\right)$ (see below).

Theorem 3.1. Let $A: S \rightarrow S$ be continuous. Suppose

(i) $F(A)$ is nonempty;

(ii $\left.{ }_{\mathrm{uw}}\right)$ for each $x \in S$, and each $p \in F(A)$, one has

$$
d(A(x), p) \leqq d(x, p) ;
$$

(iii) for each $x \in S$, one has

$$
\lim _{m \rightarrow \infty} d\left(A^{m+1}(x), A^{m}(x)\right)=0 .
$$

Let $x \in S$. Then, either $\left\{A^{m}(x)\right\}_{m=1}^{\infty}$ contains no convergent subsequence, or $\lim _{m \rightarrow \infty} A^{m}(x)$ exists, and belongs to $F(A)$.

Proof. This proof follows from the proof of Theorem 3, in almost the same way as the proof of Theorem 2.1 followed from that of Theorem 2 .

REMARK 13. In case $S$ is a Banach space, it follows from Theorem 3.1 that $\mathscr{L}(x)$ is either empty or a single element, so that the sequence $\left\{A^{m}(x)\right\}_{m=1}^{\infty}$ either has no (strongly) convergent subsequence or it converges (strongly) to an element of $F(A)$. It is of interest to notice that, under the two additional assumptions

(a) for each $x, y \in S$, one has $\|A(x)-A(y)\| \leqq\|x-y\|$;

(b) the function $I-A$, where $I$ is the identity, maps bounded closed subsets of $S$ into closed subsets of $S$.

Browder and Petryshyn [5, p. 574, Theorem 6] have proved that the sequence $\left\{A^{m}(x)\right\}_{m=1}^{\infty}$ converges (strongly) to an element of $F(A)$. That is to say, in these circumstances the possibility that $\mathscr{L}(x)$ is empty is excluded.

In order to exclude the possibility that $\mathscr{L}(x)$ may be empty in Theorems 2.1 and 3.1, one needs to assume something along the lines of Browder and Petryshyn's condition (b). The purpose of the following lemma is to give a sufficient condition which guarantees that $\mathscr{L}(x) \neq \varnothing$.

Lemma. Let $A: S \rightarrow S$ be continuous. Suppose

(iii) for each $x \in S$, one has

$$
\lim _{m \rightarrow \infty} d\left(A^{m+1}(x), A^{m}(x)\right)=0 ;
$$

(iv) the (continuous) real-valued function $f$, defined by $f(x)=d(A(x), x)$ for $x \in S$, has the property that $f$ maps bounded closed subsets of $S$ into closed sets of real numbers.

Then, for $x \in S$, whenever the sequence $\left\{A^{m}(x)\right\}_{m=1}^{\infty}$ is bounded, the set $\mathscr{L}(x)$ is nonempty. 
Proof. Suppose $\left\{A^{m}(x)\right\}_{m=1}^{\infty}$ is bounded. Then the closure of this sequence, $\mathrm{cl}\left(\left\{A^{m}(x)\right\}_{m=1}^{\infty}\right)$, is also bounded. Hence, by (iv), the set of real numbers $\operatorname{cl}\left(f\left(\left\{A^{m}(x)\right\}_{m=1}^{\infty}\right)\right)$ is closed. However, from (iii), one has

$$
\lim _{m \rightarrow \infty} d\left(A^{m+1}(x), A^{m}(x)\right)=\lim _{m \rightarrow \infty} f\left(A^{m}(x)\right)=0,
$$

so that the number 0 belongs to the set $\operatorname{cl}\left(f\left(\left\{A^{m}(x)\right\}_{m=1}^{\infty}\right)\right)$. But

which gives

$$
\operatorname{cl}\left(\left\{A^{m}(x)\right\}_{m=1}^{\infty}\right)=\left\{A^{m}(x)\right\}_{m=1}^{\infty} \cup \mathscr{L}(x),
$$

$$
\operatorname{cl}\left(f\left(\left\{A^{m}(x)\right\}_{m=1}^{\infty}\right)\right)=f\left(\left\{A^{m}(x)\right\}_{m=1}^{\infty}\right) \cup f(\mathscr{L}(x)) .
$$

Since $0 \in \operatorname{cl}\left(f\left(\left\{A^{m}(x)\right\}_{m=1}^{\infty}\right)\right)$, then either $0 \in f\left(\left\{A^{m}(x)\right\}_{m=1}^{\infty}\right)$, in which case $A^{k}(x)$ $\in F(A)$ for some $1 \leqq k<\infty$ and $\mathscr{L}(x)$ consists of the single point $A^{k}(x)$, or else $0 \in f(\mathscr{L}(x))$, in which case $\mathscr{L}(x)$ cannot be empty.

Under the hypotheses of either Theorem 2.1 or Theorem 3.1 it follows that, for $x \in S$, the sequence of iterates $\left\{A^{m}(x)\right\}_{m=1}^{\infty}$ is bounded. Hence, putting together the results of Theorem 2.1 and the lemma, and of Theorem 3.1 and the lemma, one obtains the following two theorems.

THEOREM 2.2. Suppose the hypotheses of Theorem 2.1, and hypothesis (iv) of the lemma, hold. Then the conclusion of Theorem 2.1 holds, with the addendum that $\mathscr{L}(x)$ is nonempty.

THEOREM 3.2. Suppose the hypotheses of Theorem 3.1, and hypothesis (iv) of the lemma, hold. Let $x \in S$. Then $\lim _{m \rightarrow \infty} A^{m}(x)$ exists and belongs to $F(A)$.

Suppose that the metric space $S$ is, in particular, a Banach space $B$. Theorem 3.2 specializes to the following:

TheOREM 3.3. Let $A: B \rightarrow B$ be continuous, where $B$ is a nonempty Banach space. Suppose

(i) $F(A)$ is nonempty;

(iii $\left.{ }_{\text {uw }}\right) x \in B, p \in F(A)$, implies $\|A(x)-p\| \leqq\|x-p\|$;

(iii) $x \in B$ implies that $\lim _{m \rightarrow \infty}\left\|A^{m+1}(x)-A^{m}(x)\right\|=0$;

(iv) the (continuous) real-valued function $f$, defined by $f(x)=\|x-A(x)\|$ for $x \in B$, maps bounded closed subsets of $B$ into closed sets of real numbers.

Then, for $x \in S, \lim _{m \rightarrow \infty} A^{m}(x)$ exists and belongs to $F(A)$.

The conclusion of Theorem 3.3 is the same as that of Theorem 6 of Browder and Petryshyn [5, p. 574]. Hypotheses (i) and (iii) of Theorem 3.3 coincide with the corresponding hypotheses of Theorem 6 of [5]. However, hypothesis $\left(\mathrm{ii}_{\mathrm{uw}}\right.$ ) is weaker than the corresponding assumption in [5], because Browder and Petryshyn assume that $\|A(x)-A(y)\| \leqq\|x-y\|$ for all $x, y \in B$. On the other hand, hypothesis (iv) is stronger than the corresponding assumption in [5] (see hypothesis $\left(\mathrm{iv}_{\mathrm{w}}\right)$ below and Remark 14). 
The next theorem shows that, while retaining the weaker hypothesis $\left(\mathrm{ii}_{\mathrm{uw}}\right)$ of Theorem 3.3, the hypothesis (iv) of Theorem 3.3 can be weakened to that of Theorem 6 of [5], without altering the conclusion of Theorem 3.3.

TheOREM 3.4. Let $A: B \rightarrow B$ be continuous, where $B$ is a nonempty Banach space. Suppose

(i) $F(A)$ is nonempty;

(ii $\left.{ }_{\text {uw }}\right) x \in B, p \in F(A)$, implies $\|A(x)-p\| \leqq\|x-p\|$;

(iii) $x \in B$ implies that

$$
\lim _{m \rightarrow \infty}\left\|A^{m+1}(x)-A^{m}(x)\right\|=0
$$

$\left(\mathrm{iv}_{\mathrm{w}}\right)$ the function $I-A$ (where $I$ is the identity function on $B$ ) maps bounded closed subsets of $B$ into closed subsets of $B$.

Then, for $x \in S, \lim _{m \rightarrow \infty} A^{m}(x)$ exists and belongs to $F(A)$.

Proof. The proof is identical to the proof of Theorem 6 of [5], since only ( $\mathrm{ii}_{\mathrm{uw}}$ ) is used in the argument there.

REMARK 14. It will now be shown that hypothesis (iv) of Theorem 3.3 implies hypothesis $\left(\mathrm{iv}_{\mathrm{w}}\right)$, of Theorem 3.4. Suppose, in accordance with (iv), that $f$ maps bounded closed subsets of $B$ into closed sets of real numbers. Let $C$ be a bounded closed set in $B$. Then, by (iv), the set $f(C)=\{\|(I-A)(x)\| \mid x \in C\}$ is a closed set of real numbers. But, the norm function is a continuous function on $B$ to the real numbers, while the set $f(C)$, by (iv), is a closed set of real numbers. Consequently, the inverse image of the set $f(C)$, with respect to the norm function, namely, the set $(I-A)(C)$, must be a closed subset of $B$. But this means that every bounded closed set $C$ is mapped by $I-A$ into a closed set, which is just hypothesis $\left(\mathrm{iv}_{\mathrm{w}}\right)$ of Theorem 3.4.

It is of some interest that the hypothesis of asymptotic regularity, together with the assumption that $A(S)$ is compact, enables one to prove a theorem whose conclusion is that of Theorem 2, save for the fact that $\mathscr{L}(x)$ cannot now be empty. This observation is formalized in the next theorem.

THEOREM 6. Let $A: S \rightarrow S$ be continuous, where $S$ is a nonempty metric space. Suppose

(a) $A(S)$ is compact;

(b) for each $x \in S$, one has $\lim _{m \rightarrow \infty} d\left(A^{m+1}(x), A^{m}(x)\right)=0$.

Then, for $x \in S$, the set $\mathscr{L}(x)$ is a nonempty closed and connected subset of $F(A)$. Either $\mathscr{L}(x)$ contains exactly one point or it contains uncountably many points. In case $\mathscr{L}(x)$ is just one point, then $\lim _{m \rightarrow \infty} A^{m}(x)$ exists and belongs to $F(A)$. In case $\mathscr{L}(x)$ is uncountable, then it is contained in the boundary of $F(A)$.

Proof. (1) The sequence of iterates $\left\{A^{m}(x)\right\}_{m=1}^{\infty}$ is a subset of the compact set $A(S)$. Therefore, $\mathscr{L}(x)$ is nonempty. 
(2) $\mathscr{L}(x) \subseteq F(A)$. This follows from (b), and the continuity of $A$, as in the proof of Theorem 2.1 .

(3) $\mathscr{L}(x)$ is closed. This follows as in the proof of Theorem 2.

(4) $\mathscr{L}(x)$ is connected. Let $S_{1}, S_{2}, S_{1}^{+}$, and $S_{2}^{+}$, be as in part 4 of the proof of Theorem 2. Then, $d\left(S_{1}^{+}, S_{2}^{+}\right)>0$, as before.

It will next be shown, by contradiction, that

$$
\lim _{m \rightarrow \infty} d\left(A^{m}(x), S_{1}^{+} \cup S_{2}^{+}\right)=0 .
$$

For, suppose that this limit were not zero, then there would exist $\varepsilon>0$ and a subsequence of iterates $\left\{A^{m_{i}}(x)\right\}_{i=1}^{\infty}$ such that

$$
d\left(A^{m_{i}}(x), S_{1}^{+} \cup S_{2}^{+}\right) \geqq \varepsilon>0
$$

for all $i=1,2, \ldots$ The subsequence of iterates $\left\{A^{m_{i}}(x)\right\}_{i=1}^{\infty}$ is a subset of the.compact set $A(S)$. Hence, there is a convergent subsequence of $\left\{A^{m_{i}}(x)\right\}_{i=1}^{\infty}$, which, for convenience, will again be denoted by $\left\{A^{m_{i}}(x)\right\}_{i=1}^{\infty}$. Then

$$
\lim _{i \rightarrow \infty} d\left(A^{m_{i}}(x), S_{1}^{+} \cup S_{2}^{+}\right)=d\left(\lim _{i \rightarrow \infty} A^{m_{i}}(x), S_{1}^{+} \cup S_{2}^{+}\right) \geqq \varepsilon>0 .
$$

But $\lim _{i \rightarrow \infty} A^{m_{i}}(x) \in \mathscr{L}(x) \subseteq S_{1}^{+} \cup S_{2}^{+}$, which is a contradiction.

Thus, it has been shown that

$$
\lim _{m \rightarrow \infty} d\left(A^{m}(x), S_{1}^{+} \cup S_{2}^{+}\right)=0 ;
$$

while, from hypothesis (b), one has

$$
\lim _{m \rightarrow \infty} d\left(A^{m+1}(x), A^{m}(x)\right)=0 .
$$

The remainder of the proof is exactly that of the last paragraph of part 4 of the proof of Theorem 2 .

(5) $\mathscr{L}(x)$ a single point or uncountable. This follows as in part 5 of the proof of Theorem 2.

(6) $\mathscr{L}(x)$ consists of just one point implies that $\lim _{m \rightarrow \infty} A^{m}(x)$ exists. Suppose $\mathscr{L}(x)$ consists only of the single point $p$, but that, contrary to what one wishes to prove, the sequence $\left\{A^{m}(x)\right\}_{m=1}^{\infty}$ does not converge to $p$. Then there is an $\varepsilon>0$ and a subsequence $\left\{A^{m_{i}}(x)\right\}_{i=1}^{\infty}$ such that

$$
d\left(A^{m_{i}}(x), p\right) \geqq \varepsilon>0
$$

for $i=1,2, \ldots$ The sequence $\left\{A^{m_{i}}(x)\right\}_{i=1}^{\infty}$ is a subset of the compact set $A(S)$, hence there is a convergent subsequence of $\left\{A^{m_{1}}(x)\right\}_{i=1}^{\infty}$, which, for convenience, will again be denoted by $\left\{A^{m_{i}}(x)\right\}_{i=1}^{\infty}$. Then

$$
\lim _{i \rightarrow \infty} d\left(A^{m_{i}}(x), p\right)=d\left(\lim _{i \rightarrow \infty} A^{m_{i}}(x), p\right) \geqq \varepsilon>0 .
$$


But then the two points $\lim _{i \rightarrow \infty} A^{m_{i}}(x)$ and $p$ both belong to $\mathscr{L}(x)$, which is a contradiction.

(7) $\mathscr{L}(x)$, if uncountable, lies in the boundary of $F(A)$. This follows as in part 7 of the proof of Theorem 2.

REMARK 15. Hypothesis (a), that $A(S)$ is compact, may be replaced by the weaker hypothesis that there exists a positive integer $k$ such that $A^{k}(S)$ is compact.

\section{BIBLIOGRAPHY}

1. S. Banach, Sur les opérations dans les ensembles abstraits et leur application aux équations intégrales (Thèse, Université de Léopol [=Lwów], 1920), Fund. Math. 3 (1922), 133-181.

2. M. Edelstein, On fixed and periodic points under contractive mappings, J. London Math. Soc. 37 (1962), 74-79.

3. - A remark on a theorem of M. A. Krasnoselski, Amer. Math. Monthly 73 (1966), 509-510.

4. F. E. Browder and W. V. Petryshyn, The solution by iteration of linear functional equations in Banach spaces, Bull. Amer. Math. Soc. 72 (1966), 566-570.

5. - The solution by iteration of nonlinear functional equations in Banach spaces, Bull. Amer. Math. Soc. 72 (1966), 571-575.

6. F. Tricomi, Lectures on the use of special functions by calculations with electronic computers, Lecture Series No. 47, Inst. Fluid Dynamics and Appl. Math., Univ. of Maryland, College Park, 1966.

7. -_ Un teorema sulla convergenza delle successioni formate delle successive iterate di una funzione di una variabile reale, Giorn. Mat. Battaglini 54 (1916), 1-9.

8. A. M. Ostrowski, Solution of equations and systems of equations, Academic Press, New York, 1960.

9. M. A. Krasnosel'skil, Two remarks on the method of successive approximations, Uspehi Mat. Nauk 10 (1955), no. 1(63), 123-127. (Russian)

10. V. M. Fridman, The method of successive approximations for Fredholm integral equations of the first kind, Uspehi Mat. Nauk 11 (1956), no. 1(67), 233-234. (Russian)

11. R. L. Moore, Foundations of point set theory, Amer. Math. Soc. Colloq. Publ., Vol. 13, Amer. Math. Soc., Providence, R. I., 1932.

12. S. C. Chu and R. D. Moyer, On continuous functions, commuting functions, and fixed points, Fund. Math. 59 (1966), 91-95.

13. S. Mazur, Über die kleinste konvexe Menge, die eine gegebene kompakte Menge enthält, Studia Math. 2 (1930), 7-9.

14. F. E. Browder, Nonlinear elliptic boundary value problems, Bull. Amer. Math. Soc. 69 (1963), 862-874.

15. G. J. Minty, On a " monotonicity" method for the solution of nonlinear equations in Banach spaces, Proc. Nat. Acad. Sci. U.S.A. 50 (1963), 1038-1041.

RensSelaer Polytechnic Institute, TROY, NeW YORK

UNIVERSITY OF CALIFORNia, Riverside, CALIForNia 